\title{
Effect of Static Magnetic Fields on Proliferation and Apoptosis of Gallbladder Cancer Cells: An in vitro and in vivo study
}

Huan Yang ${ }^{1,2}$, Junxi Xiang ${ }^{1,2}$, Jian Dong ${ }^{1,2}$, Jiwen Cheng ${ }^{2,3}$, Xinglong Zheng ${ }^{1,2}$, Peng Liu ${ }^{1,2}$, Rongqian Wu ${ }^{1,2}$ and Yi Lv $^{1,2^{*}}$

${ }^{1}$ Department of Hepatobiliary Surgery, First Affiliated Hospital of Xi'an Jiaotong University, China

${ }^{2}$ Institute of Advanced Surgical Technology and Engineering, First Affiliated Hospital of Xi'an Jiaotong University, Xi'an, Shaanxi Province, China

${ }^{3}$ Department of Pediatric Surgery, Second Affiliated Hospital of Xi'an Jiaotong University, China

"Corresponding author: Yi Lv, Department of Hepatobiliary Surgery, First Affiliated Hospital, Xi'an Jiaotong University, West Yanta Road, Xi'an 710061, Shaanxi Province, China, Tel: +8613201539590; E-mail: luyi169@126.com

Received date: February 17, 2018; Accepted date: February 27, 2018; Published date: March 2, 2018

Copyright: @ 2018 Yang H, et al. This is an open-access article disturbed under the terms of the Creative Commons Attribution License, which permits unrestricted use, distribution, and reproduction in any medium, provided the original author and source are created.

\begin{abstract}
Objectives: The knowledge on how static magnetic fields (SMF) of magnetic implants affect living cells and tissues is limited while these magnetic devices are more and more frequently adopted in certain surgical approaches.

Methods: In this study, we exposed cultures of human gallbladder cancer cells to SMF continuously for up to 7 days, trying to simulate the exposure pattern of surgical magnetic implants, and implanted magnetic devices into tumor-bearing nude mice for 1 month, then evaluated the effect on proliferation and apoptosis of cancer cells and tissues.

Results: It showed that after SMF exposure, whereas in vitro study showed decreased cell proliferation and increased apoptosis of the gallbladder cancer cells, in vivo study showed no significant differences both on proliferation and on apoptosis.

Conclusions: Our findings indicate that with current magnetic biliary anastomosis devices, the SMF from magnetic implants shows little effects on proliferative and apoptotic activities of gallbladder cancer cells in vivo, while there are still possibilities that long-term continuous SMF exposure from surgical magnetic implants may interact with living cells, probably in a feeble manner.
\end{abstract}

Keywords Static magnetic field; Gallbladder cancer; Magnetic implant; Proliferation; Apoptosis

\section{Introduction}

There is an increasing interest in using static magnetic devices in surgical situations like biliary or vascular anastomosing [1-3] and gastro-esophageal reflux disease [4]. The feasibility and safety of magnetic compression anastomosis have been experimentally and clinically verified, and it is considered as quick, safe and equivalent or superior to anastomoses created by traditional suture or staple techniques [1,5-7], and hence been increasingly adopted in animal experiments and in clinical practices [7-10].

Although the effects of static magnetic fields (SMF) on cells and tissues have been studied for decades, there is still limited knowledge on this topic $[11,12]$. A large number of studies have been conducted in an effort to detect biological responses, including in vitro, in vivo, human volunteer and population studies. Moreover, for different endpoints in vitro, including cell orientation, cell growth, cell metabolic activity, cell membrane physiology, and gene expression, positive and negative findings have been reported [12-15].

While most studies focused on effects of relatively short time exposure (a few hours to 1 day) or repetitive exposure [12-24], particularly strong SMF $(>1 \mathrm{~T})$ to investigate the influence of MRI, there are only limited reports on effects of long-term continuous exposure to SMF. Orthodontic magnets and implants are tools sometimes used nowadays in dental and oral medicine. Yamaguchi et al. [25] exposed gingival fibroblast to $0.2 \mathrm{~T}$ and for 6-8 months without an effect on cell growth, and Bondemark et al. [26,27] examined the effects of orthodontic magnets on the oral mucosa, the dental pulp and the gingival in a total of 15 patients which showed no histological detectable changes. Determine how continuous SMF exposure affects internal organ tissues, and neoplasm recurrence post-operatively, is essential to evaluate the effects and safety of those magnetic implants which would stay in patients' bodies for days or longer [1].

In this study, we simulated the SMF of the biliary magnetic compression anastomosis device, and evaluated the effects of SMF exposure on gallbladder cancer cells both in vitro and in vivo, as Gallbladder cancer is the most common malignancy in the biliary tract [28] and biliary reconstruction is one of the major situations magnetic anastomosis techniques and magnetic implants are used.

\section{Materials and Methods}

\section{Cell lines and cell culture}

The human primary gallbladder carcinoma cell line SGC-996 was obtained from the Academy of Life Science, Tongji University (Shanghai, China) [29] and maintained in RPMI-1640 medium 
(HyClone, South Logan, Utah, USA) supplemented with $10 \%$ fetal bovine serum and $1 \%$ penicillin-streptomycin (Gibco, Grand Island, NY, USA) at $37^{\circ} \mathrm{C}$ under a humidified atmosphere containing $5 \% \mathrm{CO}_{2}$.

\section{Magnetic field exposure in vitro}

We measured the flux density of the field from coupled magnetic compression anastomosis device for biliary anastomosis at different distance, which showed an average of $370 \mathrm{mT}$ at the pole surface (the compression surface), $72.8 \mathrm{mT}$ at $1 \mathrm{~mm}$ aside, $2.4 \mathrm{mT}$ at $30 \mathrm{~mm}$ aside. Measurement from other study [5] reports surface fields of approximately $300-600 \mathrm{mT}$ of this kind of device matches our results which shows the device designs are similar.

To simulate the flux density range, we placed the culture plates at different distances from a cuboid neodymium magnet with size of $50 \times$ $50 \times 25 \mathrm{~mm}$ (Figure 1) [16]. The flux densities of exposure of 4 exposure groups, naming as $\mathrm{m} 1$ to $\mathrm{m} 4$, are $76.4 \pm 7.5 \mathrm{mT}, 45.6 \pm 3.6 \mathrm{mT}$, $20.1 \pm 1.1 \mathrm{mT}, 2.2 \pm 0.1 \mathrm{mT}$ respectively.

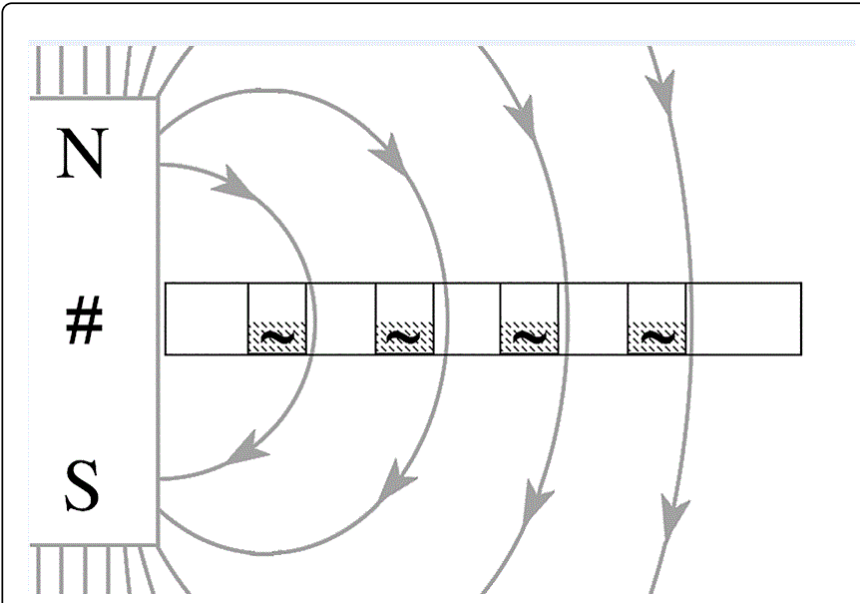

Figure 1: Scheme of the approach to simulate the magnetic field of the magnetic compression anastomosis device in vitro. \#: Magnet. $\sim:$ Cell cultures. Arrows: Lines of magnetic flux.

The flux densities of the field were measured by gaussmeter (HT20 digital gaussmeter, Hengtong Ltd., Shanghai, China).

\section{Cell proliferation}

Cell proliferation was assessed by a colorimetric procedure with the Cell Counting Kit-8 (Dojindo, Kumamoto, Japan) [30]. Cell was seeded into 96-well plates (1000 cells/well), and the cell viability was assayed every other day for 1 week after seeding. For CCK- 8 assay, cells were incubated with CCK- 8 reagent for 1 hour at $37^{\circ} \mathrm{C}$, according to the manufacturer's protocol, and detected the absorbance at $450 \mathrm{~nm}$ using a Varioskan Flash Spectral Scanning Multimode Reader (Thermo Scientific, Waltham, Massachusetts, USA).

\section{Cell apoptosis}

Cells undergoing apoptosis at the indicated time were identified using Annexin V-FITC apoptosis detection kit (KeyGEN BioTECH, Nanjing, China), following the manufacturer's instructions. In brief, the cells were exposed to SMF for 5 days, collected by centrifugation, re-suspended in binding buffer, incubated with Annexin V-FITC and propidium iodide at room temperature for $5 \mathrm{~min}$ in the dark, and then analyzed by flow cytometer (BD FACSCanto II Flow Cytometer, BD Bioscience, Franklin Lakes, New Jersey, USA) [31].

\section{In vivo experiments}

4 week-old male athymic BALB/c nude mice (Centre of Laboratory Animals, The Medical College of Xi'an Jiaotong University, Xi'an, China) were used to establish the nude mouse xenograft model. 30 mice were evenly divided into 3 groups at random: control group, sham exposure group and exposure group. SGC-996 cells were orthotopically inoculated in nude mice as described by Egberts $[32,33]$. Briefly, SGC-996 cells were suspended in FBS-free medium and mixed with equal volume of Matrigel (BD Bioscience, Franklin Lakes, New Jersey, USA) at a final concentration of $107 \mathrm{cells} / \mathrm{ml}$ and stored on ice. The nude mice were anesthetized and laparotomy was performed. The gallbladder was exposed and the bile inside aspirated. $30 \mathrm{~mL}$ of cell suspension aforementioned was injected into the gallbladder and solidified in about 30s. Then a neodymium magnet cylinder size of $\Phi 6$ $\mathrm{mm} \times 8 \mathrm{~mm}$ with biocompatible coating was fixed to the abdominal wall next to the gallbladder with 6-0 silk (Figure 2). The magnets were magnetized for exposure group, and demagnetized for sham exposure group. No magnets were implanted in control group mice. Finally, all layers of abdominal wall were closed with 6-0 silk.

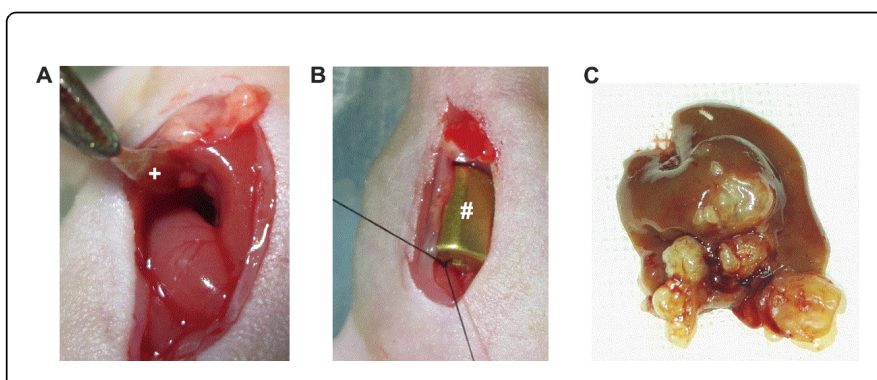

Figure 2: Nude mouse xenograft model. A) Operation site after inoculation of cancer cells in Matrigel-medium into the gallbladder. B) Fixing magnet to the abdominal wall. C) Harvested tumor tissue. +: Gallbladder. \#: Magnet.

All mice were sacrificed after 4 weeks following the surgery. The xenograft tumor tissues were harvested for pathological examination. All in vivo protocols were approved by the Institutional Animal Care and Use Committee of Xi'an Jiaotong University.

\section{Microscopic examination}

The tumor tissues were fixed in phosphate-buffered formaldehyde, embedded in paraffin, sliced into sections, and stained with hematoxylin and eosin (HE).

Immunohistochemical analysis of proliferating cell nuclear antigen (PCNA) was performed [34] with biotin conjugate anti-PCNA monoclonal antibody (Invitrogen, Waltham, Massachusetts, USA). The number of PCNA-positive cells was counted in five high-power fields selected at random, and the PCNA labeling index for each field was calculated as the percent of PCNA-positive cells (relative to the total).

Apoptosis in tumor cells was detected using the terminal deoxynucleotidyl transferase dUTP nick end labeling (TUNEL) assay [34] with In Situ Cell Death Detection Kit (Roche, Basel, Switzerland). In the same manner as PCNA, five high-power fields were selected at 
random, and the apoptotic index of each field was calculated as the percent of TUNEL-positive cells.

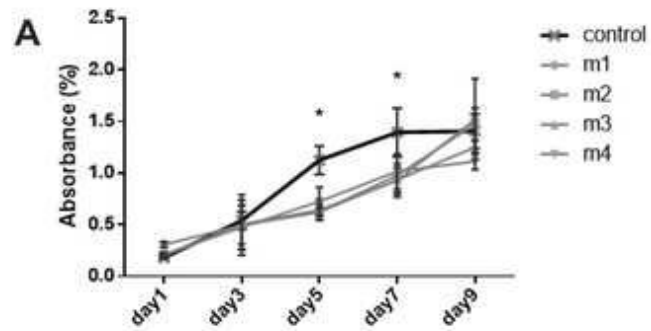

B

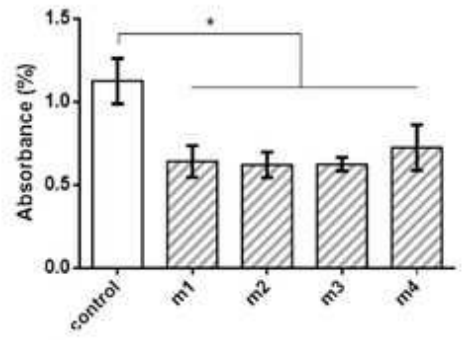

C
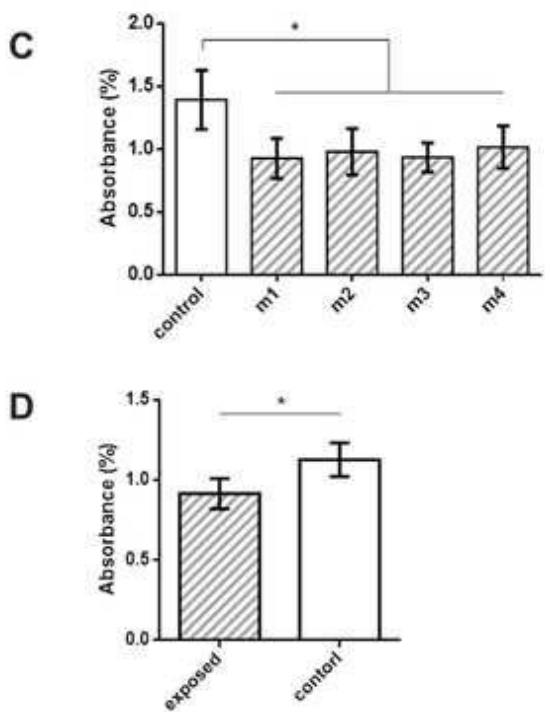

Figure 3: Cell proliferation of SGC-996 cells decreased after magnetic field exposure in vitro. A, Growth curve of each group. Cell proliferative activities shows significantly decrease on day 5 (B) and day 7 (C). D, Cell proliferation at $450 \mathrm{mT}$ (maximal flux density on surface of the magnet) on day $5^{\star}: \mathrm{p}<0.001$.

\section{Statistical Analysis}

Results are presented as mean \pm standard deviation (SD). The statistic differences were evaluated by one-way ANOVA and Student's t-test with SPSS Statistics 23.0 and GraphPad Prism 6. $\mathrm{P}<0.05$ was considered as statistically significant.

\section{Results}

\section{Cell proliferation of SGC-996 cells decreased after SMF exposure in vitro}

To assess the effect of SMF on cell proliferation, SGC-996 cells were exposed to SMF continuously of different magnetic flux density and cell proliferation assays were performed every other day for 7 days using CCK-8 assay. The results showed that proliferation of SGC-996 cells exposed to SMF significant decreased (ANOVA: all $\mathrm{p}<0.001$ on day 5 and 7, Figure 3B and 3C) compare to control while there were no significant differences in groups of different magnetic flux density. As showed in Figure 3A, while control group had a typical growth curve reached maximal cell count at day 7 , the ones of exposure groups are lower, which indicates less cell counts and lower proliferative activity.

We also exposed SGC-996 cells to higher magnetic flux density (450 $\mathrm{mT}$ ) to test the extreme situation of the cells next to magnetic pole. The results are similar after 5-day exposure (t test: $\mathrm{p}<0.001$ ) (Figure $3 \mathrm{D}$ ).

\section{Apoptosis of SGC-996 cells increased after SMF exposure in vitro}

To further evaluate the effect of SMF on SGC-996 cells in vitro, cell apoptosis was assessed. The results showed that the SMF-exposed $\mathrm{m} 1$ group had slightly but statistically significantly higher percentage of apoptotic cells than control group ( $t$ test: $\mathrm{p}<0.001$ ) (Figure 4 ).
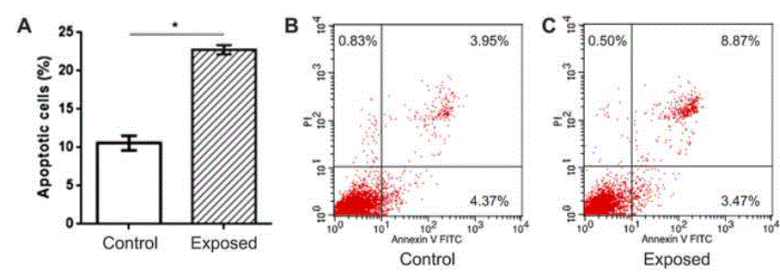

Figure 4: Magnetic field exposed group has significantly higher percentage of apoptotic cells on day 5. A: Total percentage (early apoptosis + late apoptosis) of apoptotic cells of each group. B and C: Flow cytometric analysis of apoptosis ${ }^{\star}: \mathrm{p}<0.001$.

\section{In vivo study showed no significant differences both on proliferation and on apoptosis}

The time of magnets removal reported in clinical studies ranges from 7 to 40 days for magnetic bilioenteric anastomosis and 9 to 74 days for magnetic biliobiliary anastomosis [7]. In this study, we sacrificed the mice in 28 days to guarantee tumor model establishment and meet the exposure time.

At the time of sacrifice, macroscopically visible gallbladder tumors were present with direct liver invasion in all groups. The tumors in all groups were histopathologically similar. Both the PCNA labeling indices and apoptotic indices showed no differences between each groups (ANOVA: all $\mathrm{p}>0.1$ ) (Figure 5). 


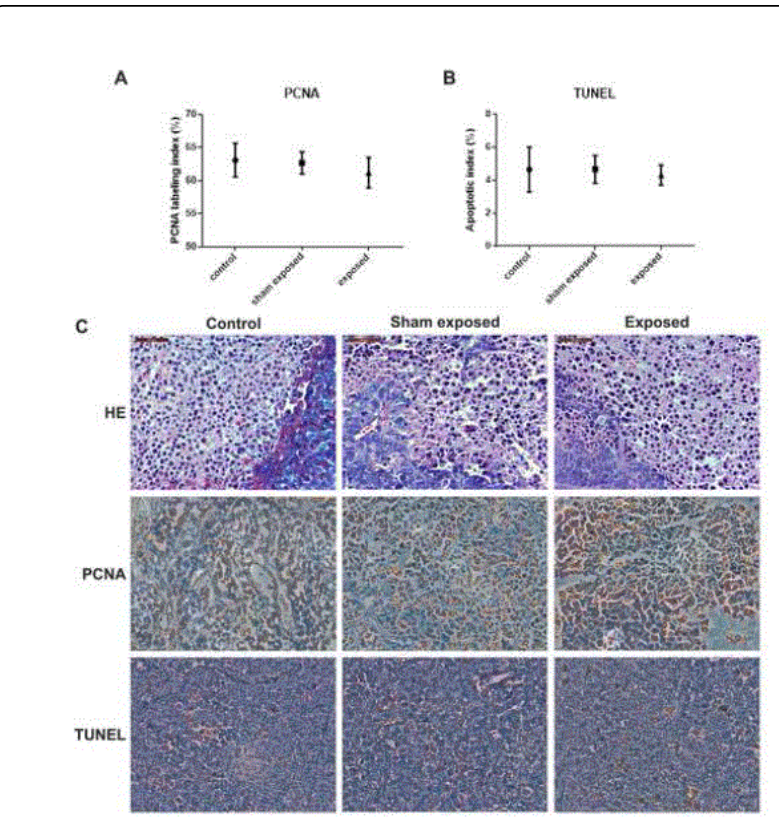

Figure 5: In vivo study showed no significant differences on PCNA labelling indices (A) and apoptotic indices (B). Microscopic view of each group with above-mentioned staining and labelling $(\mathrm{C})$.

\section{Discussion}

Magnetic surgical implants are increasingly used clinically, while there are still limit knowledge about their effects on cells and tissues especially on tumor sufferers post-operatively from the magnetic field. To our best knowledge, this is the first time we focused on how longterm continuous SMF exposure from these devices affects gallbladder cancer cells on its proliferative and apoptotic activity both in vitro and in vivo.

The proliferation of gallbladder cancer cell line could be inhibited and apoptosis be increased in SMF in vitro have been shown in this present study, but the mechanism is still unknown. There are reports on the magnetic saturation effect, also known as window-effect or threshold response, in different studies [17,18,35-37]. When flux density reaches a threshold, some effects of SMF on certain cells saturate and stop change with flux density increasing, and the reported threshold could be as low as $60 \mu \mathrm{T}$ on endothelial cells [18]. In this study, cell proliferation showed no significant differences in the groups exposed to SMF ranging from $2 \mathrm{mT}$ to $70 \mathrm{mT}$, which could be another example of window-effect, with the threshold unknown, as it could be much lower than the SMF we employed. As the mechanism how SMF affect cells is poorly understood, the molecular details of the windoweffect is still unclear, while some studies showed free radical mechanism may be involved.

Unexpectedly the results from animal experiment disagree with the in vitro tests, which need to be carefully looked into. One of the abiding weaknesses of in vitro experiments is that they fail to replicate the precise cellular conditions of an organism. This has been discussed abundantly [38-41]. In general, cells in vitro share a different environment, including cytokines and hormones, extracellular matrix, host immune response, etc., with ones in vivo, which may lead to different responses to stimuli.

Another possible interference is the spatial pattern of SMF gradient. Even though the SMFs we employed in vitro and in vivo were of similar flux density range and both were with gradients, the spatial patterns of the gradients were different. There are reports on gradient and uniform SMFs caused different responses in cells and tissues [20-23], which could due to magneto mechanical effects and/or other possible mechanisms [12]. It is not clear that whether altered pattern of SMF gradient could also make a difference, which is beyond the scope of this study and needs further investigation.

There are also some other possible factors, like cells may have adapted to the stimuli after the even longer exposure, or respiratory movement of the animals may cause periodic positional changes of the magnets, which were located next to the diaphragm, and hence altered the static nature of SMF. All these needs further investigation to determine whether and how they may influence the biological behaviours.

In conclusion, our findings indicate that with current magnetic biliary anastomosis devices, SMF from the magnetic implants shows little effects on proliferative and apoptotic activities of gallbladder cancer cells in vivo, while in vitro study showed alteration in cell activities, and there are some possibilities that long-term continuous SMF exposure from surgical magnetic implants may interact with living cells, probably in a feeble manner though. Current studies have shown that different patterns of SMF exposure can alter a variety of biological activities on different levels, in a quite unpredictable way when it comes to certain cell or tissue [12-14,42]. Considering some techniques, like vessel anastomosis with magnetic devices, require a permanent implantation [43-45], determining whether even longer SMF exposure may leads to different consequences would also be essential.

\section{Acknowledgements}

The research was supported by the National Natural Science Foundation of China (81470896 and 81501608).

\section{References}

1. Muraoka N, Uematsu H, Yamanouchi E, Kinoshita K, Takeda T, et al (2005) Yamanouchi magnetic compression anastomosis for bilioenteric anastomotic stricture after living-donor liver transplantation. J Vasc Interv Radiol 16: 1263-1267.

2. Liu SQ, Lei P, Cao ZP, Lv Y, Li JH, et al. (2012) Nonsuture anastomosis of arteries and veins using the magnetic pinned-ring device: a histologic and scanning electron microscopic study. Ann Vasc Surg 26: 985-995.

3. Liu SQ, Lei P, Cui XH, Lv Y, Li JH, et al. (2013) Sutureless anastomoses using magnetic rings in canine liver transplantation model. J Surg Res 185: 923-933.

4. Ganz RA, Peters JH, Horgan S, Bemelman WA, Dunst CM, et al. (2013) Esophageal sphincter device for gastroesophageal reflux disease. $\mathrm{N}$ Engl J Med 368: 719-727.

5. Jamshidi R, Stephenson JT, Clay JG, Pichakron KO, Harrison MR, et al. (2009) Magnamosis: magnetic compression anastomosis with comparison to suture and staple techniques. J Pediatr Surg 44: 222-228.

6. Mimuro A, Tsuchida A, Yamanouchi E, Itoi T, Ozawa T, et al. (2003) A novel technique of magnetic compression anastomosis for severe biliary stenosis. Gastrointest Endosc 58: 283-287.

7. Jang SI, Choi J, Lee DK, (2015) Magnetic compression anastomosis for treatment of benign biliary stricture. Dig Endosc 27: 239-249. 
8. Xue F, Guo HC, Li JP, Lu JW, Wang HH, et al. (2016) Choledochojejunostomy with an innovative magnetic compressive anastomosis: How to determine optimal pressure? World J Gastroenterol 22: 2326-2335.

9. Oya H, Sato Y, Yamanouchi E, Yamamoto S, Hara Y, et al. (2012) Magnetic compression anastomosis for bile duct stenosis after donor left hepatectomy: a case report. Transplant Proc 44: 806-809.

10. Zaritzky M, Ben R, Johnston K (2014) Magnetic gastrointestinal anastomosis in pediatric patients. J Pediatr Surg 49: 1131-1137.

11. Tenforde TS (1979) Magnetic Field Effect on Biological Systems. Plenum Press.

12. WHO-World Health Organization (2006) Environmental Health Criteria 232, Static Fields., Geneva.

13. SCENIHR-Scientific Committee on Emerging and Newly Identified Health Risks (2009) Health Effects of Exposure to EMF.

14. (2009) International Commission on Non-Ionizing Radiation, P., Guidelines on limits of exposure to static magnetic fields. Health Phys 96: 504-514.

15. Miyakoshi J (2005) Effects of static magnetic fields at the cellular level. Prog Biophys Mol Biol 87: 213-223.

16. Chionna A, Tenuzzo B, Panzarini E, Dwikat MB, Abbro L, et al. (2005) Time dependent modifications of Hep G2 cells during exposure to static magnetic fields. Bioelectromagnetics 26: 275-286.

17. Martino CF (2011) Static magnetic field sensitivity of endothelial cells. Bioelectromagnetics 32: 506-508.

18. Martino CF, Perea H, Hopfner U, Ferguson VL, Wintermantel E, et al. (2010) Effects of weak static magnetic fields on endothelial cells. Bioelectromagnetics 31: 296-301.

19. McDonald F (1993) Effect of static magnetic fields on osteoblasts and fibroblasts in vitro. Bioelectromagnetics 14: 187-196.

20. Okano H, Onmori R, Tomita N, Ikada Y, et al. (2006) Effects of a moderate-intensity static magnetic field on VEGF-A stimulated endothelial capillary tubule formation in vitro. Bioelectromagnetics 27: 628-640.

21. Okano H, Tomita N, Ikada Y, (2007) Effects of $120 \mathrm{mT}$ static magnetic field on TGF-betal-inhibited endothelial tubular formation in vitro. Bioelectromagnetics 28: 497-499.

22. Okano H, Tomita N, Ikada Y (2008) Spatial gradient effects of $120 \mathrm{mT}$ static magnetic field on endothelial tubular formation in vitro. Bioelectromagnetics 29: 233-236.

23. Wang Z, Yang P, Xu H, Qian A, Hu L, et al. (2009) Inhibitory effects of a gradient static magnetic field on normal angiogenesis. Bioelectromagnetics 30: 446-453.

24. Wiskirchen J, Grönewäller EF, Heinzelmann F, Kehlbach R, Rodegerdts E, et al. (2000) Human fetal lung fibroblasts: in vitro study of repetitive magnetic field exposure at 0.2, 1.0, and 1.5 T. Radiology 215: 858-862.

25. Yamaguchi H, Hosokawa K, Soda A, Miyamoto H, Kinouchi Y, et al. (1993) Effects of seven months' exposure to a static 0.2 T magnetic field on growth and glycolytic activity of human gingival fibroblasts. Biochim Biophys Acta 1156: 302-306.

26. Bondemark L, Kurol J, Wisten A (1995) Extent and flux density of static magnetic fields generated by orthodontic samarium-cobalt magnets. Am J Orthod Dentofacial Orthop 107: 488-496.
27. Bondemark L, Kurol J, Larsson A (1998) Long-term effects of orthodontic magnets on human buccal mucosa--a clinical, histological and immunohistochemical study. Eur J Orthod 20: 211-218.

28. Diehl AK (1980) Epidemiology of gallbladder cancer: a synthesis of recent data. J Natl Cancer Inst 65: 1209-1214.

29. Yang YQ, Tao Hh, Yang HC (2003) Establishing of Human Primary Gallbladder Cancer Cell Line SGC-996. J Tongji University (Medical Science) 24: 457-459.

30. Ishiyama M, Miyazono Y, Sasamoto K, Ohkura Y, Ueno K, et al. (1997) A highly water-soluble disulfonated tetrazolium salt as a chromogenic indicator for $\mathrm{NADH}$ as well as cell viability. Talanta 44: 1299-1305.

31. Zhang G, Gurtu V, Kain SR, Yan G, et al. (1997) Early detection of apoptosis using a fluorescent conjugate of annexin V. Biotechniques 23: 525-31.

32. Egberts JH, Schniewind B, Schafmayer C, Kruse ML, Sipos B, et al. (2007) Establishment of a novel orthotopic xenograft model of human gallbladder carcinoma. Clin Exp Metastasis 24: 141-148.

33. Du Q, Jiang L, Wang X, Wang M, She F, et al. (2014) Tumor necrosis factor-alpha promotes the lymphangiogenesis of gallbladder carcinoma through nuclear factor-kappaB-mediated upregulation of vascular endothelial growth factor-C. Cancer Sci 105: 1261-1271.

34. Mita Y, Ajiki T, Kamigaki T, Okazaki T, Hori H, et al. (2007) Antitumor effect of gemcitabine on orthotopically inoculated human gallbladder cancer cells in nude mice. Ann Surg Oncol 14: 1374-1380.

35. Volpe P, (2003) Interactions of zero-frequency and oscillating magnetic fields with biostructures and biosystems. Photochem Photobiol Sci 2: 637-648.

36. Belyaev I, Alipov YD, Harms-Ringdahl M (1997) Effects of zero magnetic field on the conformation of chromatin in human cells. Biochim Biophys Acta 1336: 465-473.

37. Binhi VN, Alipov YD, Belyaev IY (2001) Effect of static magnetic field on E. coli cells and individual rotations of ion-protein complexes. Bioelectromagnetics 22: 79-86.

38. Lorian V (1989) In vitro simulation of in vivo conditions: physical state of the culture medium. J Clin Microbiol 27: 2403-2406.

39. Zitvogel L, Tursz T (2005) In vivo veritas. Nat Biotechnol 23: 1372-1374.

40. Sica A, Mantovani A (2012) Macrophage plasticity and polarization: in vivo veritas. J Clin Invest 122: 787-795.

41. Fazekas de St Groth B, Smith AL, Higgins CA (2004) T cell activation: in vivo veritas. Immunol Cell Biol 82: 260-268.

42. Rosen AD (2003) Mechanism of action of moderate-intensity static magnetic fields on biological systems. Cell Biochem Biophys 39: 163-173.

43. Erdmann D, Sweis R, Heitmann C, Yasui K, Olbrich KC, et al. (2004) Side-to-side sutureless vascular anastomosis with magnets. J Vasc Surg 40: 505-511.

44. Filsoufi F, Farivar RS, Aklog L, Anderson CA, Chen RH, et al. (2004) Automated distal coronary bypass with a novel magnetic coupler (MVP system). J Thorac Cardiovasc Surg 127: 185-192.

45. Heitmann C, Khan FN, Erdmann D, Olbrich KC, Adam Sharkawy A, et al. (2007) Vein graft anastomoses with magnets. J Plast Reconstr Aesthet Surg 60: 1296-1301. 